\section{OOPHORECTOMY FOR CANCER OF THE BREAST.}

ONE of the most remarkable facts in surgery - if it be a fact-and one which is entirely unexplained, however many may be the hypotheses put forward in regard to it, is the effect of oophorectomy upon cancer of the breast.

There are many difficulties in the way of coming to any definite conclusion as to the utility of this proceeding, the great and standing one being that, in the present state of our knowledge, and in view of the probability of being able to give long, if not permanent, relief by removing the breast and its cancer altogether whenever it is seen in an early stage, no one would feel justified in undertaking a purely speculative operation like oophorectomy in early and therefore hopeful cases. Hence, it is only in the inoperatable and otherwise hopeless cases that this operation can be done, and one need not, therefore, be surprised that the results shown are not altogether encouraging. But it must be remarked that the extraordinary improvement in some cases, and the fact that any improvement at all should be found to occur in others, are sufficiently surprising, and go far to encourage a hope, not exactly that oophorectomy may be found to cure, but that its success may give the clue to the direction in which cure is to be sought. Mr. Stanley Boyd has recently reviewed the whole subject and collected certain details concerning forty-one cases in which this operation has been done by various surgeons, thirteen of these having been operated on by Mr. Stanley Boyd himself, and one by Mrs. Boyd. Summing up the matter, he says "that oophorectomy should be offered in cases, other than the very acute, in women over forty, with no visceral or bony lesions, in fair condition, and before the menopause. He adds.that the work must still be experimental, and that while the surgeon must decide what it is right to do in each case, the patient or the patient's friends should understand the experimental nature of the treatment. $\mathrm{He}$ would combine the oophorectomy with removal of the breast in cases in which the disease was in such an advanced form that he could not hope for prolonged immunity from a local operation. It should be noted that in the hands of several surgeons the administration of thyroid extract has been combined with the operation.

\section{THE RESTING POSITION OF ANOPHELES.}

In is always well to correct error at once, for the makers of medical books have a bad habit of copying one from another, so that an error once set agoing may pass on through many generations of handbooks and encyclopædias. Major Donald Ross and Mr. A. E. Austen in the report of their expedition to Sierra Leone in 1899 laid great stress on the position assumed by mosquitoes when at rest, as a means of discriminating between the malaria-bearing mosquitoes of the genus Anopheles and those of the genus Culex, the latter of which so far seem to take no:part in the dissemination of human malaria. They said that the Anopheles always, when at rest, sat in a characteristic: attitude, their bodies being maintained at an angle of at least $45^{\circ}$ to the surface on which they settled. Frequently, they said, the tips of the palpi were in contact with the wall on which the insect was resting, and the body was so much elevated that it was practically at right angles to the wall, and the insect appeared as if it were standing upon its head, and it was held that, owing to this extremely characteristic resting attitude of Anopheles, it would be impossible for anyone who had once seen a specimen at rest to mistake it for a Culex, for the latter. when at rest. allows its body to hang down vertically.

Now Dr. Sambon and Dr. Low ${ }^{1}$ have been passing the summer on the Campagna, and they directly traverse this statement as to the attitude of these insects. They say that they have observed thousands of Anopheles, but none of them ever assumed the position described by the members of the Liverpool Malarial Mission, and they hold that although the resting position may be a means of distinguishing between certain species, it is not a criterion by which Anopheles can be distinguished from Culex, which was the point insisted on, a point which has already been copied into many text books.

1 Brit. Med. Jour., Oct. 20.

\title{
PROGRESS OF SURGERY.
}

\section{SURGERY OF THE LIVER AND GALL-} BLADDER.

Neoplasms of the Iiver are rarely seen early enough to allow of their removal. The adenomata and a few carcinomatous deposits-either primary, or secondary by continuity of tissue alone-are the only malignant tumours ever likely to be considered operable. It is only in such cases in which there is distinct delimitation of the disease that we can hope to do anything of a radical nature, and it is also evident that as disease approaches the hilus it becomes more dangerous and less suitable to any operative treatment. Experience has shown that the cautery and ligature, alone or combined, are the safest and quickest means of controlling bleeding from hepatic tissue, and that pressure with packing is an useful adjuvant. The cauterised wound in the liver should be treated by packing, and the abdominal cavity protected from contamination by the same means. All means of combating the effects of hæmorrhage should be ready for immediate use, for it is never certain that we can avoid serious trouble in this regard. There is no prospect that any but growths $\mathbf{n}$ the superficial portions of the liver can be attacked successfully, even supposing that it were possible to make a diagnosis with reasonable certainty when the tumour lies very deep. Bile from healthy liver tissue will not hurt the peritoneum, but from inflamed and infected gallducts it will cause peritonitis. ${ }^{1}$

Cholelithiasis.-Richardson ${ }^{2}$ says gall-stones must be removed as soon as diagnosed. The indications for operation consist in finding facetted stones in the stools, nonfacetted stones so found contra-indicating operation. Repeated attacks with or without finding stones points to operation. Quiescence no more contra-indicates operation than it does in appendicitis. Repeated attacks of -tenderness and persistent pain over the gall-bladder are among the most valuable indications. Kehr ${ }^{3}$ has performed operations for gall-stones in 491 cases during the past ten years, the mortality being from 2 to 7 per cent. Some patients continue to complain of colicky pain after operation. These troubles, he thinks, are due to calculi left in the gall-bladder because they are not found during the operation, and are never caused by the formation of fresh calculi. Kehr therefore suggests the name of "spurious relapses" to the pairs originating in 